Acta Crystallographica Section E

Structure Reports

Online

ISSN 1600-5368

William T. A. Harrison, ${ }^{\text {a* }}$ S. L. Gaonkar, ${ }^{b}$ H. G. Anilkumar ${ }^{b}$ and H. S. Yathirajan ${ }^{b}$

a Department of Chemistry, University of Aberdeen, Meston Walk, Aberdeen AB24 3UE, Scotland, and ${ }^{\mathbf{b}}$ Department of Studies in Chemistry, University of Mysore, Manasagangotri, Mysore 570 006, India

Correspondence e-mail:

w.harrison@abdn.ac.uk

\section{Key indicators}

Single-crystal X-ray study

$T=120 \mathrm{~K}$

Mean $\sigma(\mathrm{C}-\mathrm{C})=0.004 \AA$

$R$ factor $=0.041$

$w R$ factor $=0.079$

Data-to-parameter ratio $=17.0$

For details of how these key indicators were automatically derived from the article, see http://journals.iucr.org/e.

\title{
5-Bromo-1-(4-fluorophenyl)-1,3-dihydro- isobenzofuran
}

The title compound, $\mathrm{C}_{14} \mathrm{H}_{10} \mathrm{BrFO}$, possesses normal geometrical parameters. The dihedral angle between the two ring systems is $71.50(9)^{\circ}$. An unusually short intermolecular Br. . Br contact of 3.4311 (5) ^ occurs.

\section{Comment}

The title compound, (I), is an intermediate in the synthesis of the antidepressant drug citalopram (Liechti et al., 2000). More generally, phthalans show distinctive redox chemistry (Azzena et al., 1996). We have previously deposited (CSD-260624; Cambridge Structural Database; Allen, 2002) data for a poor quality structure from a twinned crystal of (I).<smiles>Fc1ccc(C2OCc3cc(Br)ccc32)cc1</smiles>

(I)

The geometrical parameters for (I) are normal. Each molecule of (I) is chiral (the arbitrarily chosen asymmetric unit has an $S$ conformation at $\mathrm{C} 7$ ), but crystal symmetry generates a racemic mixture of the two enantiomers. The ninemembered isobenzofuran ring system (C7-C14/O1) is almost planar [r.m.s. deviation from the mean plane $=0.018 \AA$; maximum $=0.038$ (3) $\AA$ for C14] and the dihedral angle between the two ring systems (C7-C14/O1 and $\mathrm{C} 1-\mathrm{C} 6)$ is $71.50(9)^{\circ}$.

A PLATON (Spek, 2003) analysis of (I) identified two possible $\mathrm{C}-\mathrm{H} \cdots \mathrm{F}$ interactions (Table 1 ) that may help to stabilize the crystal packing (Fig. 2). There are no significant $\pi-\pi$ stacking interactions in (I).

Inversion symmetry generates a short intermolecular $\operatorname{Br} 1 \cdots \mathrm{Br} 1^{\mathrm{i}}$ [symmetry code: (i) $2-x,-y, 1-z$ ] separation of 3.4311 (5) $\AA$ which is significantly less than the van der Waals contact distance of $3.70 \AA$ for two $\mathrm{Br}$ atoms (Bondi, 1964). Some workers have ascribed specific attractive forces to such short intermolecular halogen-halogen contacts (Desiraju \& Parthasarathy, 1989). A database survey of such contacts by Eriksson \& Hu (2001) shows that the present separation lies at the lower end of the observed range of intermolecular $\mathrm{Br} \cdots \mathrm{Br}$ distances. However, these workers are less certain of the nature of such contacts, and suggest that they may be the consequence - rather than the cause - of the crystal packing. 
In the related 1-(4-fluorophenyl)-1,3-dihydroisobenzofuran-5-carbonitrile [i.e. where a cyanide group replaces the $\mathrm{Br}$ atom in (I)], there are two molecules in the asymmetric unit with distinctly different degrees of twist between their ring systems (Yathirajan et al., 2004).

\section{Experimental}

5-Bromo-3H-isobenzofuran-1-one (2.13 g, $10 \mathrm{mmol})$ was subjected to a Grignard reaction with 4-fluorophenyl magnesium bromide $(2.4 \mathrm{~g}$, $12 \mathrm{mmol})$ in tetrahydrofuran $(10 \mathrm{ml})$ at $273 \mathrm{~K}$. The resulting product was treated with sodium borohydride $(0.37 \mathrm{~g}, 10 \mathrm{mmol})$ in methanol $(10 \mathrm{ml})$ to obtain the diol, which was cyclized with $p$-toluene sulfonic acid $(1 \mathrm{~g}, 5.81 \mathrm{mmol})$ in toluene $(10 \mathrm{ml})$ at $353 \mathrm{~K}$, yielding crude $(\mathrm{I})$. Diffraction-quality crystals were obtained by recrystallization from $n$ hexane (Bigler et al., 1977) (m.p. 318 K).

\section{Crystal data}

$$
\begin{aligned}
& \mathrm{C}_{14} \mathrm{H}_{10} \mathrm{BrFO} \\
& M_{r}=293.13 \\
& \text { Monoclinic, } P 2_{b} / c \\
& a=6.0560(3) \AA \\
& b=7.8659(4) \AA \\
& c=24.2289(14) \AA \\
& \beta=92.542(3)^{\circ} \\
& V=1153.03(11) \AA^{3} \\
& Z=4
\end{aligned}
$$

$$
\begin{aligned}
& D_{x}=1.689 \mathrm{Mg} \mathrm{m}^{-3} \\
& \text { Mo } K \alpha \text { radiation } \\
& \text { Cell parameters from } 2707 \\
& \quad \text { reflections } \\
& \theta=1.0-27.5^{\circ} \\
& \mu=3.56 \mathrm{~mm}^{-1} \\
& T=120(2) \mathrm{K} \\
& \text { Block, yellow } \\
& 0.24 \times 0.15 \times 0.10 \mathrm{~mm}
\end{aligned}
$$

\section{Data collection}

Nonius KappaCCD diffractometer $\omega$ and $\varphi$ scans

Absorption correction: multi-scan

(SADABS; Bruker, 2003)

$T_{\min }=0.482, T_{\max }=0.718$

12389 measured reflections

2630 independent reflections

\section{Refinement}

Refinement on $F^{2}$

$R\left[F^{2}>2 \sigma\left(F^{2}\right)\right]=0.041$

$w R\left(F^{2}\right)=0.079$

$S=1.02$

2630 reflections

155 parameters

$\mathrm{H}$-atom parameters constrained

$$
\begin{aligned}
& 1742 \text { reflections with } I>2 \sigma(I) \\
& R_{\text {int }}=0.072 \\
& \theta_{\max }=27.6^{\circ} \\
& h=-7 \rightarrow 7 \\
& k=-10 \rightarrow 10 \\
& l=-31 \rightarrow 27
\end{aligned}
$$

$$
\begin{gathered}
w=1 /\left[\sigma^{2}\left(F_{\mathrm{o}}{ }^{2}\right)+(0.0257 P)^{2}\right. \\
+0.4294 P] \\
\text { where } P=\left(F_{\mathrm{o}}{ }^{2}+2 F_{\mathrm{c}}{ }^{2}\right) / 3 \\
(\Delta / \sigma)_{\max }<0.001 \\
\Delta \rho_{\max }=0.56 \mathrm{e} \AA^{-3} \\
\Delta \rho_{\min }=-0.40 \AA^{-3}
\end{gathered}
$$

Extinction correction: SHELXL97 Extinction coefficient: 0.0023 (6)

\section{Table 1}

Hydrogen-bond geometry $\left(\AA{ }^{\circ}\right)$.

\begin{tabular}{lllll}
\hline$D-\mathrm{H} \cdots A$ & $D-\mathrm{H}$ & $\mathrm{H} \cdots A$ & $D \cdots A$ & $D-\mathrm{H} \cdots A$ \\
\hline $\mathrm{C} 9-\mathrm{H} 9 \cdots \mathrm{F} 1^{\mathrm{i}}$ & 0.95 & 2.54 & $3.324(4)$ & 140 \\
$\mathrm{C} 14-\mathrm{H} 14 B \cdots \mathrm{F} 1^{\mathrm{ii}}$ & 0.99 & 2.52 & $3.265(4)$ & 132 \\
\hline
\end{tabular}

Symmetry codes: (i) $-x, y-\frac{1}{2},-z+\frac{1}{2}$; (ii) $-x+1, y+\frac{1}{2},-z+\frac{1}{2}$.

The $\mathrm{H}$ atoms were positioned geometrically, with $\mathrm{C}-\mathrm{H}=0.95-$ $0.99 \AA$, and refined as riding, with $U_{\text {iso }}(\mathrm{H})=1.2 U_{\text {eq }}$ (carrier).

Data collection: COLLECT (Nonius, 1998); cell refinement: SCALEPACK (Otwinowski \& Minor, 1997); data reduction: SCALEPACK and DENZO (Otwinowski \& Minor, 1997), and SORTAV (Blessing, 1995); program(s) used to solve structure: SHELXS97 (Sheldrick, 1997); program(s) used to refine structure:

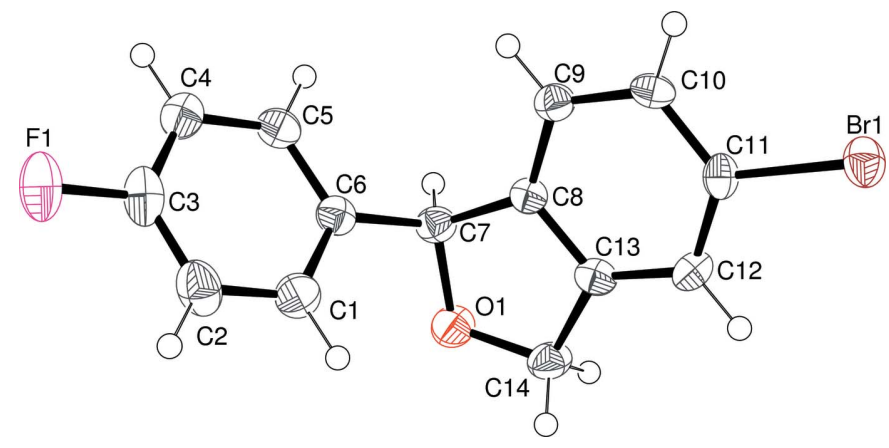

Figure 1

View of (I), showing $50 \%$ displacement ellipsoids and arbitrary spheres for the $\mathrm{H}$ atoms.

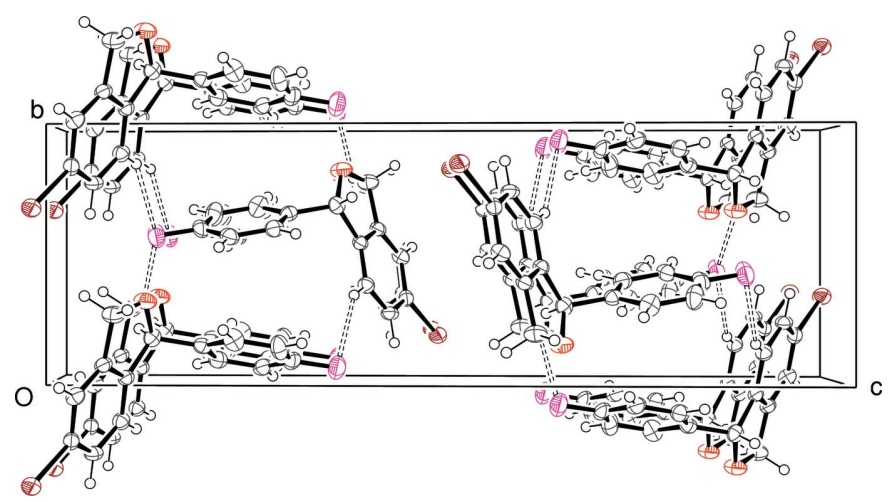

Figure 2

Unit-cell packing in (I), viewed down [100], showing 50\% displacement ellipsoids and arbitrary spheres for the $\mathrm{H}$ atoms, with short $\mathrm{C}-\mathrm{H} \cdots \mathrm{F}$ interactions shown as dashed lines.

SHELXL97 (Sheldrick, 1997); molecular graphics: ORTEP-3 (Farrugia, 1997); software used to prepare material for publication: SHELXL97.

We thank the EPSRC National Crystallography Service for data collection. HGA thanks the University of Mysore for provision of research facilities.

\section{References}

Allen, F. H. (2002). Acta Cryst. B58, 380-388.

Azzena, U., Demartis, S. \& Melloni, G. (1996). J. Org. Chem. 61, 4913-4919.

Bigler, A. J., Bogeso, K. B. \& Toft, A. (1977). Eur. J. Med. Chem. 12, 289-295.

Blessing, R. H. (1995). Acta Cryst. A51, 33-38.

Bondi, A. (1964). J. Phys. Chem. 68, 441-451.

Bruker (2003). SADABS. Bruker AXS Inc., Madison, Wisconsin, USA.

Desiraju, G. R. \& Parthasarathy, R. (1989). J. Am. Chem. Soc. 111, 8725-8726.

Eriksson, L. \& Hu, J. (2001). Acta Cryst. E57, o930-0932.

Farrugia, L. J. (1997). J. Appl. Cryst. 30, 565.

Liechti, M. E., Baumann, C., Gamma, A. \& Vollenweider, F. X. (2000). Neuropsychopharmacology, 22, 513-521.

Nonius (1998). COLLECT. Nonius BV, Delft, The Netherlands.

Otwinowski, Z. \& Minor, W. (1997). Methods in Enzymology, Vol. 276, Macromolecular Crystallography, Part A, edited by C. W. Carter Jr \& R. M. Sweet, pp. 307-326. New York: Academic Press.

Sheldrick, G. M. (1997). SHELXS97 and SHELXL97. University of Göttingen, Germany.

Spek, A. L. (2003). J. Appl. Cryst. 36, 7-13.

Yathirajan, H. S., Nagaraj, B., Gaonkar, S. L., Narasegowda, R. S., Nagaraja, P. \& Bolte, M. (2004). Acta Cryst. E60, 2225-2227. 


\title{
supporting information
}

Acta Cryst. (2006). E62, o1534-o1535 [https://doi.org/10.1107/S1600536806009810]

\section{5-Bromo-1-(4-fluorophenyl)-1,3-dihydroisobenzofuran}

\author{
William T. A. Harrison, S. L. Gaonkar, H. G. Anilkumar and H. S. Yathirajan
}

5-Bromo-1-(4-fluorophenyl)-1,3-dihydroisobenzofuran

Crystal data

$\mathrm{C}_{14} \mathrm{H}_{10} \mathrm{BrFO}$

$M_{r}=293.13$

Monoclinic, $P 2_{1} / c$

Hall symbol: $-\mathrm{P} 2 \mathrm{ybc}$

$a=6.0560(3) \AA$

$b=7.8659(4) \AA$

$c=24.2289(14) \AA$

$\beta=92.542(3)^{\circ}$

$V=1153.03(11) \AA^{3}$

$Z=4$

\section{Data collection}

Nonius KappaCCD diffractometer

Radiation source: fine-focus sealed tube Graphite monochromator $\omega$ and $\varphi$ scans

Absorption correction: multi-scan

(SADABS; Bruker, 2003)

$T_{\min }=0.482, T_{\max }=0.718$

\section{Refinement}

Refinement on $F^{2}$

Least-squares matrix: full

$R\left[F^{2}>2 \sigma\left(F^{2}\right)\right]=0.041$

$w R\left(F^{2}\right)=0.079$

$S=1.02$

2630 reflections

155 parameters

0 restraints

Primary atom site location: structure-invariant direct methods

Secondary atom site location: difference Fourier map
$F(000)=584$

$D_{\mathrm{x}}=1.689 \mathrm{Mg} \mathrm{m}^{-3}$

Mo $K \alpha$ radiation, $\lambda=0.71073 \AA$

Cell parameters from 2707 reflections

$\theta=1.0-27.5^{\circ}$

$\mu=3.56 \mathrm{~mm}^{-1}$

$T=120 \mathrm{~K}$

Block, yellow

$0.24 \times 0.15 \times 0.10 \mathrm{~mm}$

12389 measured reflections

2630 independent reflections

1742 reflections with $I>2 \sigma(I)$

$R_{\text {int }}=0.072$

$\theta_{\max }=27.6^{\circ}, \theta_{\min }=3.4^{\circ}$

$h=-7 \rightarrow 7$

$k=-10 \rightarrow 10$

$l=-31 \rightarrow 27$

Hydrogen site location: inferred from neighbouring sites

$\mathrm{H}$-atom parameters constrained

$w=1 /\left[\sigma^{2}\left(F_{\mathrm{o}}^{2}\right)+(0.0257 P)^{2}+0.4294 P\right]$

where $P=\left(F_{\mathrm{o}}{ }^{2}+2 F_{\mathrm{c}}{ }^{2}\right) / 3$

$(\Delta / \sigma)_{\max }<0.001$

$\Delta \rho_{\max }=0.56 \mathrm{e} \AA^{-3}$

$\Delta \rho_{\min }=-0.40$ e $\AA^{-3}$

Extinction correction: SHELXL97, $\mathrm{Fc}^{*}=\mathrm{kFc}\left[1+0.001 \mathrm{xFc}^{2} \lambda^{3} / \sin (2 \theta)\right]^{-1 / 4}$

Extinction coefficient: 0.0023 (6) 


\section{Special details}

Geometry. All e.s.d.'s (except the e.s.d. in the dihedral angle between two 1.s. planes) are estimated using the full covariance matrix. The cell e.s.d.'s are taken into account individually in the estimation of e.s.d.'s in distances, angles and torsion angles; correlations between e.s.d.'s in cell parameters are only used when they are defined by crystal symmetry. An approximate (isotropic) treatment of cell e.s.d.'s is used for estimating e.s.d.'s involving 1.s. planes.

Refinement. Refinement of $F^{2}$ against ALL reflections. The weighted $R$-factor $w R$ and goodness of fit $S$ are based on $F^{2}$, conventional $R$-factors $R$ are based on $F$, with $F$ set to zero for negative $F^{2}$. The threshold expression of $F^{2}>\sigma\left(F^{2}\right)$ is used only for calculating $R$-factors (gt) etc. and is not relevant to the choice of reflections for refinement. $R$-factors based on $F^{2}$ are statistically about twice as large as those based on $F$, and $R$ - factors based on ALL data will be even larger.

Fractional atomic coordinates and isotropic or equivalent isotropic displacement parameters $\left(\AA^{2}\right)$

\begin{tabular}{lllll}
\hline & $x$ & $y$ & $z$ & $U_{\text {iso }} * / U_{\text {eq }}$ \\
\hline C1 & $0.3615(5)$ & $0.6855(5)$ & $0.26210(15)$ & $0.0366(9)$ \\
H1 & 0.5049 & 0.7253 & 0.2731 & $0.044^{*}$ \\
C2 & $0.3091(6)$ & $0.6559(5)$ & $0.20662(15)$ & $0.0420(9)$ \\
H2 & 0.4144 & 0.6748 & 0.1794 & $0.050^{*}$ \\
C3 & $0.1012(6)$ & $0.5986(4)$ & $0.19235(14)$ & $0.0357(9)$ \\
C4 & $-0.0572(6)$ & $0.5688(4)$ & $0.22975(14)$ & $0.0335(8)$ \\
H4 & -0.2002 & 0.5291 & 0.2183 & $0.040^{*}$ \\
C5 & $-0.0007(5)$ & $0.5990(4)$ & $0.28501(14)$ & $0.0311(8)$ \\
H5 & -0.1070 & 0.5790 & 0.3119 & $0.037^{*}$ \\
C6 & $0.2072(5)$ & $0.6577(4)$ & $0.30177(13)$ & $0.0274(7)$ \\
C7 & $0.2636(5)$ & $0.6902(4)$ & $0.36229(13)$ & $0.0276(7)$ \\
H7 & 0.1256 & 0.7199 & 0.3813 & $0.033^{*}$ \\
C8 & $0.3774(5)$ & $0.5441(4)$ & $0.39220(12)$ & $0.0232(7)$ \\
C9 & $0.3061(5)$ & $0.3792(4)$ & $0.39959(13)$ & $0.0274(8)$ \\
H9 & 0.1672 & 0.3430 & 0.3841 & $0.033^{*}$ \\
C10 & $0.4407(5)$ & $0.2669(4)$ & $0.43009(13)$ & $0.0263(8)$ \\
H10 & 0.3949 & 0.1529 & 0.4356 & $0.032 *$ \\
C11 & $0.6416(5)$ & $0.3234(4)$ & $0.45219(12)$ & $0.0258(7)$ \\
C12 & $0.7142(5)$ & $0.4893(4)$ & $0.44546(13)$ & $0.0272(8)$ \\
H12 & 0.8528 & 0.5262 & 0.4610 & $0.033^{*}$ \\
C13 & $0.5774(5)$ & $0.5985(4)$ & $0.41530(13)$ & $0.0260(7)$ \\
C14 & $0.6066(5)$ & $0.7837(4)$ & $0.40280(15)$ & $0.0334(8)$ \\
H14A & 0.6119 & 0.8516 & 0.4373 & $0.040^{*}$ \\
H14B & 0.7449 & 0.8029 & 0.3834 & $0.040^{*}$ \\
O1 & $0.4186(4)$ & $0.8294(3)$ & $0.36828(10)$ & $0.0370(6)$ \\
F1 & $0.0466(3)$ & $0.5716(3)$ & $0.13736(8)$ & $0.0472(6)$ \\
Br1 & $0.82479(5)$ & $0.17116(4)$ & $0.494648(14)$ & $0.03340(14)$ \\
& & & &
\end{tabular}

Atomic displacement parameters $\left(\AA^{2}\right)$

\begin{tabular}{lllllll}
\hline & $U^{11}$ & $U^{22}$ & $U^{33}$ & $U^{12}$ & $U^{13}$ & $U^{23}$ \\
\hline C1 & $0.0242(17)$ & $0.046(2)$ & $0.040(2)$ & $0.0044(16)$ & $0.0043(15)$ & $0.0050(18)$ \\
C2 & $0.040(2)$ & $0.056(3)$ & $0.031(2)$ & $0.0112(19)$ & $0.0092(16)$ & $0.0047(19)$ \\
C3 & $0.049(2)$ & $0.030(2)$ & $0.028(2)$ & $0.0183(17)$ & $-0.0033(18)$ & $-0.0054(16)$ \\
C4 & $0.037(2)$ & $0.026(2)$ & $0.036(2)$ & $0.0035(15)$ & $-0.0035(17)$ & $-0.0044(16)$ \\
C5 & $0.0331(19)$ & $0.0271(19)$ & $0.034(2)$ & $0.0003(15)$ & $0.0055(16)$ & $0.0012(15)$
\end{tabular}




\begin{tabular}{lllllll}
\hline C6 & $0.0274(17)$ & $0.0232(18)$ & $0.0316(19)$ & $0.0038(14)$ & $0.0022(14)$ & $0.0021(15)$ \\
C7 & $0.0250(17)$ & $0.031(2)$ & $0.0277(19)$ & $-0.0014(15)$ & $0.0053(14)$ & $-0.0019(15)$ \\
C8 & $0.0251(17)$ & $0.0210(18)$ & $0.0237(18)$ & $0.0002(14)$ & $0.0026(13)$ & $-0.0020(14)$ \\
C9 & $0.0272(17)$ & $0.027(2)$ & $0.0277(19)$ & $-0.0025(14)$ & $0.0015(14)$ & $-0.0014(14)$ \\
C10 & $0.0296(18)$ & $0.0182(17)$ & $0.032(2)$ & $-0.0031(14)$ & $0.0058(15)$ & $-0.0008(14)$ \\
C11 & $0.0278(16)$ & $0.0277(18)$ & $0.0220(17)$ & $0.0078(15)$ & $-0.0002(13)$ & $-0.0012(15)$ \\
C12 & $0.0214(16)$ & $0.028(2)$ & $0.032(2)$ & $0.0000(14)$ & $-0.0025(14)$ & $-0.0051(15)$ \\
C13 & $0.0306(18)$ & $0.0206(18)$ & $0.0270(19)$ & $-0.0013(14)$ & $0.0054(15)$ & $-0.0037(14)$ \\
C14 & $0.0291(19)$ & $0.025(2)$ & $0.046(2)$ & $-0.0034(14)$ & $-0.0020(17)$ & $0.0010(16)$ \\
O1 & $0.0387(13)$ & $0.0231(13)$ & $0.0484(16)$ & $-0.0017(11)$ & $-0.0072(11)$ & $0.0028(11)$ \\
F1 & $0.0592(14)$ & $0.0534(14)$ & $0.0284(12)$ & $0.0208(10)$ & $-0.0033(10)$ & $-0.0057(10)$ \\
Br1 & $0.0392(2)$ & $0.0276(2)$ & $0.0330(2)$ & $0.00621(16)$ & $-0.00337(14)$ & $0.00021(16)$ \\
\hline
\end{tabular}

Geometric parameters $\left(\AA,{ }^{\circ}\right)$

\begin{tabular}{|c|c|c|c|}
\hline $\mathrm{C} 1-\mathrm{C} 2$ & $1.387(5)$ & $\mathrm{C} 8-\mathrm{C} 13$ & $1.380(4)$ \\
\hline $\mathrm{C} 1-\mathrm{C} 6$ & $1.387(4)$ & $\mathrm{C} 8-\mathrm{C} 9$ & $1.381(4)$ \\
\hline $\mathrm{C} 1-\mathrm{H} 1$ & 0.9500 & $\mathrm{C} 9-\mathrm{C} 10$ & $1.392(4)$ \\
\hline $\mathrm{C} 2-\mathrm{C} 3$ & $1.367(5)$ & C9-H9 & 0.9500 \\
\hline $\mathrm{C} 2-\mathrm{H} 2$ & 0.9500 & $\mathrm{C} 10-\mathrm{C} 11$ & $1.381(4)$ \\
\hline $\mathrm{C} 3-\mathrm{C} 4$ & $1.369(5)$ & $\mathrm{C} 10-\mathrm{H} 10$ & 0.9500 \\
\hline $\mathrm{C} 3-\mathrm{F} 1$ & $1.375(4)$ & $\mathrm{C} 11-\mathrm{C} 12$ & $1.389(4)$ \\
\hline $\mathrm{C} 4-\mathrm{C} 5$ & $1.388(4)$ & $\mathrm{C} 11-\mathrm{Br} 1$ & $1.903(3)$ \\
\hline $\mathrm{C} 4-\mathrm{H} 4$ & 0.9500 & $\mathrm{C} 12-\mathrm{C} 13$ & $1.380(4)$ \\
\hline $\mathrm{C} 5-\mathrm{C} 6$ & $1.385(4)$ & $\mathrm{C} 12-\mathrm{H} 12$ & 0.9500 \\
\hline $\mathrm{C} 5-\mathrm{H} 5$ & 0.9500 & $\mathrm{C} 13-\mathrm{C} 14$ & $1.500(4)$ \\
\hline $\mathrm{C} 6-\mathrm{C} 7$ & $1.513(4)$ & $\mathrm{C} 14-\mathrm{O} 1$ & $1.428(4)$ \\
\hline $\mathrm{C} 7-\mathrm{O} 1$ & $1.445(3)$ & $\mathrm{C} 14-\mathrm{H} 14 \mathrm{~A}$ & 0.9900 \\
\hline $\mathrm{C} 7-\mathrm{C} 8$ & $1.509(4)$ & $\mathrm{C} 14-\mathrm{H} 14 \mathrm{~B}$ & 0.9900 \\
\hline $\mathrm{C} 7-\mathrm{H} 7$ & 1.0000 & & \\
\hline $\mathrm{C} 2-\mathrm{C} 1-\mathrm{C} 6$ & $120.8(3)$ & $\mathrm{C} 13-\mathrm{C} 8-\mathrm{C} 7$ & $109.4(3)$ \\
\hline $\mathrm{C} 2-\mathrm{C} 1-\mathrm{H} 1$ & 119.6 & $\mathrm{C} 9-\mathrm{C} 8-\mathrm{C} 7$ & $129.7(3)$ \\
\hline $\mathrm{C} 6-\mathrm{C} 1-\mathrm{H} 1$ & 119.6 & $\mathrm{C} 8-\mathrm{C} 9-\mathrm{C} 10$ & $119.1(3)$ \\
\hline $\mathrm{C} 3-\mathrm{C} 2-\mathrm{C} 1$ & $118.0(3)$ & $\mathrm{C} 8-\mathrm{C} 9-\mathrm{H} 9$ & 120.5 \\
\hline $\mathrm{C} 3-\mathrm{C} 2-\mathrm{H} 2$ & 121.0 & $\mathrm{C} 10-\mathrm{C} 9-\mathrm{H} 9$ & 120.5 \\
\hline $\mathrm{C} 1-\mathrm{C} 2-\mathrm{H} 2$ & 121.0 & $\mathrm{C} 11-\mathrm{C} 10-\mathrm{C} 9$ & $119.1(3)$ \\
\hline $\mathrm{C} 2-\mathrm{C} 3-\mathrm{C} 4$ & $123.6(3)$ & $\mathrm{C} 11-\mathrm{C} 10-\mathrm{H} 10$ & 120.4 \\
\hline $\mathrm{C} 2-\mathrm{C} 3-\mathrm{F} 1$ & $118.3(3)$ & $\mathrm{C} 9-\mathrm{C} 10-\mathrm{H} 10$ & 120.4 \\
\hline $\mathrm{C} 4-\mathrm{C} 3-\mathrm{F} 1$ & $118.0(3)$ & $\mathrm{C} 10-\mathrm{C} 11-\mathrm{C} 12$ & $122.3(3)$ \\
\hline $\mathrm{C} 3-\mathrm{C} 4-\mathrm{C} 5$ & $117.3(3)$ & $\mathrm{C} 10-\mathrm{C} 11-\mathrm{Br} 1$ & $119.3(2)$ \\
\hline $\mathrm{C} 3-\mathrm{C} 4-\mathrm{H} 4$ & 121.3 & $\mathrm{C} 12-\mathrm{C} 11-\mathrm{Br} 1$ & $118.3(2)$ \\
\hline $\mathrm{C} 5-\mathrm{C} 4-\mathrm{H} 4$ & 121.3 & $\mathrm{C} 13-\mathrm{C} 12-\mathrm{C} 11$ & $117.5(3)$ \\
\hline $\mathrm{C} 6-\mathrm{C} 5-\mathrm{C} 4$ & $121.5(3)$ & $\mathrm{C} 13-\mathrm{C} 12-\mathrm{H} 12$ & 121.3 \\
\hline $\mathrm{C} 6-\mathrm{C} 5-\mathrm{H} 5$ & 119.3 & $\mathrm{C} 11-\mathrm{C} 12-\mathrm{H} 12$ & 121.3 \\
\hline $\mathrm{C} 4-\mathrm{C} 5-\mathrm{H} 5$ & 119.3 & $\mathrm{C} 8-\mathrm{C} 13-\mathrm{C} 12$ & $121.1(3)$ \\
\hline $\mathrm{C} 5-\mathrm{C} 6-\mathrm{C} 1$ & $118.7(3)$ & $\mathrm{C} 8-\mathrm{C} 13-\mathrm{C} 14$ & $109.2(3)$ \\
\hline $\mathrm{C} 5-\mathrm{C} 6-\mathrm{C} 7$ & $120.3(3)$ & $\mathrm{C} 12-\mathrm{C} 13-\mathrm{C} 14$ & $129.7(3)$ \\
\hline
\end{tabular}




$\begin{array}{llll}\mathrm{C} 1-\mathrm{C} 6-\mathrm{C} 7 & 121.0(3) & \mathrm{O} 1-\mathrm{C} 14-\mathrm{C} 13 & 105.3(2) \\ \mathrm{O} 1-\mathrm{C} 7-\mathrm{C} 8 & 104.4(2) & \mathrm{O} 1-\mathrm{C} 14-\mathrm{H} 14 \mathrm{~A} & 110.7 \\ \mathrm{O} 1-\mathrm{C} 7-\mathrm{C} 6 & 110.1(2) & \mathrm{C} 13-\mathrm{C} 14-\mathrm{H} 14 \mathrm{~A} & 110.7 \\ \mathrm{C} 8-\mathrm{C} 7-\mathrm{C} 6 & 114.5(2) & \mathrm{O} 1-\mathrm{C} 14-\mathrm{H} 14 \mathrm{~B} & 110.7 \\ \mathrm{O} 1-\mathrm{C} 7-\mathrm{H} 7 & 109.2 & \mathrm{C} 13-\mathrm{C} 14-\mathrm{H} 14 \mathrm{~B} & 110.7 \\ \mathrm{C} 8-\mathrm{C} 7-\mathrm{H} 7 & 109.2 & \mathrm{H} 14 \mathrm{~A}-\mathrm{C} 14-\mathrm{H} 14 \mathrm{~B} & 108.8 \\ \mathrm{C} 6-\mathrm{C} 7-\mathrm{H} 7 & 109.2 & \mathrm{C} 14-\mathrm{O} 1-\mathrm{C} 7 & 111.5(2) \\ \mathrm{C} 13-\mathrm{C} 8-\mathrm{C} 9 & 120.8(3) & & \\ & & & -1.0(5) \\ \mathrm{C} 6-\mathrm{C} 1-\mathrm{C} 2-\mathrm{C} 3 & 0.0(5) & \mathrm{C} 13-\mathrm{C} 8-\mathrm{C} 9-\mathrm{C} 10 & -178.7(3) \\ \mathrm{C} 1-\mathrm{C} 2-\mathrm{C} 3-\mathrm{C} 4 & -0.1(5) & \mathrm{C} 7-\mathrm{C} 8-\mathrm{C} 9-\mathrm{C} 10 & 0.1(4) \\ \mathrm{C} 1-\mathrm{C} 2-\mathrm{C} 3-\mathrm{F} 1 & 178.9(3) & \mathrm{C} 8-\mathrm{C} 9-\mathrm{C} 10-\mathrm{C} 11 & 0.5(5) \\ \mathrm{C} 2-\mathrm{C} 3-\mathrm{C} 4-\mathrm{C} 5 & -0.1(5) & \mathrm{C} 9-\mathrm{C} 10-\mathrm{C} 11-\mathrm{C} 12 & 179.3(2) \\ \mathrm{F} 1-\mathrm{C} 3-\mathrm{C} 4-\mathrm{C} 5 & -179.1(3) & \mathrm{C} 9-\mathrm{C} 10-\mathrm{C} 11-\mathrm{Br} 1 & -0.2(5) \\ \mathrm{C} 3-\mathrm{C} 4-\mathrm{C} 5-\mathrm{C} 6 & 0.3(5) & \mathrm{C} 10-\mathrm{C} 11-\mathrm{C} 12-\mathrm{C} 13 & -179.0(2) \\ \mathrm{C} 4-\mathrm{C} 5-\mathrm{C} 6-\mathrm{C} 1 & -0.4(5) & \mathrm{Br} 1-\mathrm{C} 11-\mathrm{C} 12-\mathrm{C} 13 & 1.3(5) \\ \mathrm{C} 4-\mathrm{C} 5-\mathrm{C} 6-\mathrm{C} 7 & 179.7(3) & \mathrm{C} 9-\mathrm{C} 8-\mathrm{C} 13-\mathrm{C} 12 & 179.5(3) \\ \mathrm{C} 2-\mathrm{C} 1-\mathrm{C} 6-\mathrm{C} 5 & 0.2(5) & \mathrm{C} 7-\mathrm{C} 8-\mathrm{C} 13-\mathrm{C} 12 & -177.4(3) \\ \mathrm{C} 2-\mathrm{C} 1-\mathrm{C} 6-\mathrm{C} 7 & -179.9(3) & \mathrm{C} 9-\mathrm{C} 8-\mathrm{C} 13-\mathrm{C} 14 & 0.8(3) \\ \mathrm{C} 5-\mathrm{C} 6-\mathrm{C} 7-\mathrm{O} 1 & -147.9(3) & \mathrm{C} 7-\mathrm{C} 8-\mathrm{C} 13-\mathrm{C} 14 & -0.8(4) \\ \mathrm{C} 1-\mathrm{C} 6-\mathrm{C} 7-\mathrm{O} 1 & 32.2(4) & \mathrm{C} 11-\mathrm{C} 12-\mathrm{C} 13-\mathrm{C} 8 & 177.7(3) \\ \mathrm{C} 5-\mathrm{C} 6-\mathrm{C} 7-\mathrm{C} 8 & 94.9(3) & \mathrm{C} 11-\mathrm{C} 12-\mathrm{C} 13-\mathrm{C} 14 & -3.6(3) \\ \mathrm{C} 1-\mathrm{C} 6-\mathrm{C} 7-\mathrm{C} 8 & -85.0(4) & \mathrm{C} 8-\mathrm{C} 13-\mathrm{C} 14-\mathrm{O} 1 & 177.8(3) \\ \mathrm{O} 1-\mathrm{C} 7-\mathrm{C} 8-\mathrm{C} 13 & 2.3(3) & \mathrm{C} 12-\mathrm{C} 13-\mathrm{C} 14-\mathrm{O} 1 & 5.2(3) \\ \mathrm{C} 6-\mathrm{C} 7-\mathrm{C} 8-\mathrm{C} 13 & 122.7(3) & \mathrm{C} 13-\mathrm{C} 14-\mathrm{O} 1-\mathrm{C} 7 & -128.1(3) \\ \mathrm{O} 1-\mathrm{C} 7-\mathrm{C} 8-\mathrm{C} 9 & -179.7(3) & \mathrm{C} 8-\mathrm{C} 7-\mathrm{O} 1-\mathrm{C} 14 & \mathrm{C} 13) \\ \mathrm{C} 6-\mathrm{C} 7-\mathrm{C} 8-\mathrm{C} 9 & -59.3(4) & \mathrm{C} 6-\mathrm{C} 7-\mathrm{O} 1-\mathrm{C} 14 & \\ & & & \end{array}$

Hydrogen-bond geometry $\left(\AA,{ }^{\circ}\right)$

\begin{tabular}{lllll}
\hline$D-\mathrm{H} \cdots A$ & $D-\mathrm{H}$ & $\mathrm{H} \cdots A$ & $D \cdots A$ & $D-\mathrm{H} \cdots A$ \\
\hline $\mathrm{C} 9-\mathrm{H} 9 \cdots \mathrm{F} 1^{\mathrm{i}}$ & 0.95 & 2.54 & $3.324(4)$ & 140 \\
$\mathrm{C} 14-\mathrm{H} 14 B \cdots \mathrm{F} 1^{\mathrm{ii}}$ & 0.99 & 2.52 & $3.265(4)$ & 132 \\
\hline
\end{tabular}

Symmetry codes: (i) $-x, y-1 / 2,-z+1 / 2$; (ii) $-x+1, y+1 / 2,-z+1 / 2$. 